\title{
EXTRACHROMOSOMAL FACTORS AFFECTING MATING REACTIONS IN SCHIZOPHYLLUM COMMUNE
}

\author{
Y. PARAG \\ Department of Genetics, The Hebrew University, Jerusalem, Israel
}

Received 9.iv.74

\begin{abstract}
SUMmary
Extrachromosomal factors modifying mating reactions in Schizophyllum commune were found. The $P$ factor appeared spontaneously, while the $F$ factor appeared following nitrosoguanidine treatment. When a strain carrying $P$ was mated with wild-type strains, it invariably developed pseudoclamps (usually typical of common- $B$ heterokaryons), while there developed on the side of the wild-type strain the dikaryon or heterokaryon expected from the genotypes involved. In a cross $A x B x P \times A y B y$, fruiting bodies from the side of the wild type gave rise only to wild-type segregants with mating types as expected from their genotypes. A selfed fruiting body from the side of the $P$ strain gave rise to progeny which did carry the $P$ factor, but its expression was modified. It had inconsistent and symmetrical effects in $A \neq B \neq$ and $A \neq B=$ matings, but the original type of asymmetrical reaction in $A=B \neq$ matings. In the next generation the effect of $P$ was weak and symmetrical. Strains carrying $F$ factor were flat (morphology typical of mutation of the $B$ factor) and were compatible with strains with which they should be incompatible according to the genotypes, e.g. $A x B x F \times A y B x$ gave a dikaryon. The factor showed nonMendelian segregation and was extremely unstable.
\end{abstract}

\section{INTRODUGTION}

IN Schizophyllum commune, as in many other higher basidiomycetous fungi, mating compatibility is controlled by two genetic factors, $A$ and $B$, each with a long series of alternative states $(A 1, A 2, A 3$, etc., and $B 1, B 2, B 3$, etc.). The formation of dikaryons with clamp connections and fruiting requires the fusion of two homokaryons differing one from another in both the $A$ and the $B$ factors $(A \neq B \neq$ e.g. $A 1 B 1 \times A 2 B 2)$. The $A$ and the $B$ factors are each composed of two discrete genes, $\alpha$ and $\beta$, each gene with a multiple allelic series. The combination of the $\alpha$ and $\beta$ alleles determines the specificity of the $A$ and the $B$ factors. When two homokaryons carrying the same $A$ factor but different $B$ factors $(A=B \neq$ e.g. $A 1 B 1 \times A 1 B 2)$ are crossed, the result is a common-A heterokaryon with a typically aberrant morphology called flat. In $A \neq B=$ matings (e.g. $A 1 B 1 \times A 2 B 1$ ), a common- $B$ heterokaryon is formed along the region of confrontation. This heterokaryon has binucleate or uninucleate apical cells and is typified by pseudoclamps, which start to develop like clamp connections but fail to fuse with the corresponding subapical cells (Parag, 1965; Raper, 1966; Parag, 1970).

Ghanges in mating specificities followed by changes in mating reactions and in the types of the resulting heterokaryons have been found to occur as a result of mutations in the incompatibility factors (Parag, 1962a; Raper, Boyd and Raper, 1965; Raper, 1966) or by modifiers unlinked to the incompatibility factors (Raper and Raper, 1968). In two series of experiments, two apparent extrachromosomal factors have been found, which have 
a pronounced effect on mating specificity and on the heterokaryons formed following such matings. These two factors have been tentatively named $P$ factor (for pseudoclamps) and $\mathrm{F}$ factor (for flat).

\section{Materials AND MEthods}

The general methods of preparing the media, mating type tests and notations, progeny tests and mutation induction are described elsewhere (Raper and Miles, 1958; Parag, 1965; Parag, 1970). The specificities of $A$ and $B$ factors isolated from nature (Raper et al., 1958) are given by the number that follows, like $A 41, B 13$; for recombinant factors (Raper, 1966) both $\alpha$ and $\beta$ specificities are given, such as $A \alpha 4-\beta 1, B \alpha 1-\beta 2$. It is important to understand from which side of the confrontation line the tested fruiting body was isolated. Therefore, in this paper, in matings of two homokaryons, the one on the left served-or was designated as serving-as acceptor of nuclei (" "female "), while the one on the right served-or was designated as serving-as donor of nuclei (" male"). In practice, when a pair of strains is mated, the mate from which a mycelium is subsequently isolated for the test is thus the acceptor, and vice versa.

\section{Unilaterality}

A strain in a cross may serve only as a donor and not as an acceptor; such a strain is called unilateral. The result is that on the side of the normal strain there develops the mycelium expected from the mating types of the two strains (e.g. dikaryon in $A \neq B \neq$ combinations), while the unilateral strain retains its homokaryotic morphology (Raper, 1966, p. 21). Sometimes standard strains and tested segregants appear to be thin, which-probably due to a chromosomal mutation unrelated to the phenomenon studied here-has a typical morphology and is unilateral. Such strains are not therefore useful for testing reciprocality in matings.

\section{Asymmetry in matings}

The term asymmetry is used to describe a mating in which both strains are-or are expected to be-donors as well as acceptors, but each strain develops a different morphology. One of the strains develops the morphology expected from the mating type combination (e.g. dikaryon in the case of $A \neq B \neq)$, while the other strain develops a morphology unexpected from the mating type combinations (pseudoclamped mycelium in the case studied here).

\section{Di-mon matings}

In order to verify the genotypes of the two nuclear types of a dikaryon, or a suspected dikaryon, it was mated with a homokaryotic tester (di-mon matings, Papazian, 1950; Raper, 1966); either the mating reaction with the tester or the genetic analysis of the derived dikaryon indicated the genotypes of the two nuclei of the tested dikaryon (Parag, 1962b).

For convenience, the strains are frequently mentioned by their numbers, as follows:

$\begin{array}{lll}\text { B3006I, } A 41 B 2 P & 1054, A 43 B 43 & 534-40, A \alpha 2-\beta 6 B 13 \\ \text { B3006III, } A 41 B 2 P & 1093, A 47 B 47 & 534-2-3, A \alpha 3-\beta 5 B 13\end{array}$




$\begin{array}{lll}\text { B3016I, } A 41 B 2 P & 1690, A 41 B 42 \text { ad5 } & \text { C40, } A 43 B 43 \\ 699, A 41, B 41 & \text { B9020, A42 B51 arg6 } & \text { Es-84, } A \alpha 4-\beta 1 B 24 \\ 845, A 51 B 51 & \text { L328, } A 42 B 42 & \text { MS-7, } A 42 B 42 \\ 991, A 97 B 97 & \text { P207, } A 41 B 51 & \end{array}$

\section{The gytoplasmic $P$ factor}

(i) Origin and mating reactions

Three strains isolated from one cross showed very peculiar mating reactions. The cross was diploid (Parag and Nachman, 1966) $\times$ haploid:

$$
\frac{A 41 B 42 \text { ad2 nic }+}{A 42 B 42++\arg 6} \times A 41 B 2
$$

Eleven segregants including B3016 and B3006 showed the het morphology and mating reaction typical of common- $A$ heterokaryons. These heterokaryons were assumed to be disomics for the chromosome carrying the $B$ factor (Raper and Oetinger, 1962), as could readily arise in a diploid $\times$ haploid cross. It was expected that hyphal tip isolations from these apparent heterokaryons would give homokaryotic mycelia with one or the other of the $B$ factors. However, isolations from two of the flat mycelia yielded unexpected homokaryons. The isolates I and III from the flat B3006, and isolate I from the flat B3016 were found to be $A 41 B 2 P$, when $P$ denotes a factor controlling asymmetry in mating reactions as described in table 1 . These three isolates

TABle 1

Mating reactions of three strains carrying $\mathrm{P}$ factor, with four types of testers

\begin{tabular}{|c|c|c|c|c|c|}
\hline \multirow[b]{2}{*}{$P$ strain } & \multicolumn{4}{|c|}{ Testers* } & \multirow[b]{2}{*}{ Genotypes } \\
\hline & $A 41 B y$ & $A x B 2$ & $A x B y$ & $A 41 B 2$ & \\
\hline $\begin{array}{l}\text { B3006I } \\
\text { B3006III }\end{array}$ & $\begin{array}{l}\mathrm{PG} \backslash \mathrm{F} \\
\mathrm{PG} \dagger \mathrm{F} \uparrow\end{array}$ & $\begin{array}{l}\mathrm{PC} \backslash- \\
\mathrm{PC} \backslash- \\
\mathrm{PC}-\end{array}$ & $\begin{array}{l}\mathrm{PG} \backslash+ \\
\mathrm{PG} \backslash+\end{array}$ & $\begin{array}{l}\mathrm{PC} \backslash- \\
\mathrm{PG} \backslash-\end{array}$ & $\begin{array}{l}A 41 B 2 P \\
A 41 B 2 P \\
A 41 B 2 P\end{array}$ \\
\hline
\end{tabular}

* The sign to right of diagonal indicates the mating reaction on the side of the tester; the sign to left of diagonal indicates the mating reaction on the side of the tested $P$ strain; $x$ and $y$ indicate $A$ different from $A 41$ and $B$ different from $B 2$, respectively. PC, pseudoclamps; +, dikaryon with clamp connections, typical of $A \neq B \neq$ matings; $\mathrm{F}$, flat, typical of $A=B \neq$ mating; - , no change in homokaryotic morphology, typical of $A \neq B=$ and $A=B=$ matings.

$\dagger$ Results demonstrated in figs. 1-4.

developed pseudoclamps when mated with any tester regardless of its mating type, while the tester developed into the type of heterokaryon expected from the genotypes of the two partners in the mating. The mycelia with pseudoclamps had the morphology and pattern of nuclear division as in common- $B$ heterokaryons (Parag, 1965, 1970): they had either binucleate apical cells with conjugate nuclear divisions synchronised with the formation of uninucleate pseudoclamps or uninucleate apical cells with nuclear divisions synchronised with the formation of anucleate pseudoclamps. These matingtype tests were repeated many times with different testers with the same results; the unmated strains, which served as controls, retained their homokaryotic morphology during two years of experiments. 
It is assumed that since a phenotypic change on both sides of the lines of confrontation in the two types of $B \neq$ matings shown in table $I$ is found, bilateral nuclear migration occurred and the genomes of the mycelia on both sides were identical. For example in the mating B3006III, A41 B2 P $\times 699$, $A 41 \mathrm{~B} 41$, the mycelium carrying pseudoclamps developed on the side of B3006III is genotypically identical with the flat mycelium developed on the side of 699 ; both are common- $A$ heterokaryons $(A 41 B 2+A 41 B 41)$. The cause for the phenotypic difference should therefore be sought in the cytoplasm. The effect of $P$ is epistatic to the effect of the incompatibility factors, so that any homokaryotic mycelium carrying $P$ starts to carry pseudoclamps after being mated with any other mycelium, regardless of the mating-type combinations involved. To check this hypothesis it was first necessary to show that the two phenotypically different mycelia derived from the same cross were identical, as regards chromosomal genes. In other words, fruiting bodies developed on the dikaryotic mycelium should show normal segregation of mating types, while fruiting bodies developed on pseudoclamped mycelium should yield only or partly, segregants with the $P$ factor. Unfortunately, the $P$ factor apparently inhibited fruiting especially in the pseudoclamped mycelia and in dikaryons derived from mating pseudoclamped mycelia with appropriate homokaryons.

\section{(ii) Evidence against chromosomal inheritance}

Evidence is seen in table 2 that the dikaryon developed on the side of the wild-type strain also carries the nuclei of the $P$ strain, but the $P$ effect was not observed; none of the progeny from such dikaryons showed any sign of $P$ (table 2, L5, L6).

\section{(iii) Differences between reciprocal matings}

Evidence is given for migration of nuclei from each homokaryon into the other. When the wild-type strain was acceptor ("female "), this resulted in dikaryons (table 2, L5, L6). When the $P$ strain was the acceptor, the mycelium with the two nuclear types developed pseudoclamps (table 2, L4, L8). In these two experiments the pseudoclamped mycelia isolated from the side of the $P$ strain also carried nuclei of the wild-type strain. Evidence is given in L4 by di-mon mating, and in L8 by progeny tests. (L8 progeny could not be tested further; the thin characteristic meant that they could be used only as donors, and thus their response while used as acceptors could not be tested.)

\section{(iv) Reciprocal crosses}

Only indirect evidence is given for differences between progenies of reciprocal crosses, in comparisons between the crosses in experiments L5 and L6 versus L7 (table 2). When the fruiting body developed on the dikaryon on the side of the wild-type strain, the progeny showed the mating reactions expected from their genotypes (L5 and L6). When the fruiting body developed on the side of the pseudoclamped mycelium, all the progeny showed the effect of $P$, although modified (L7). However, only the $P$ strain contributed to the fruiting body which may have resulted from selfing. 


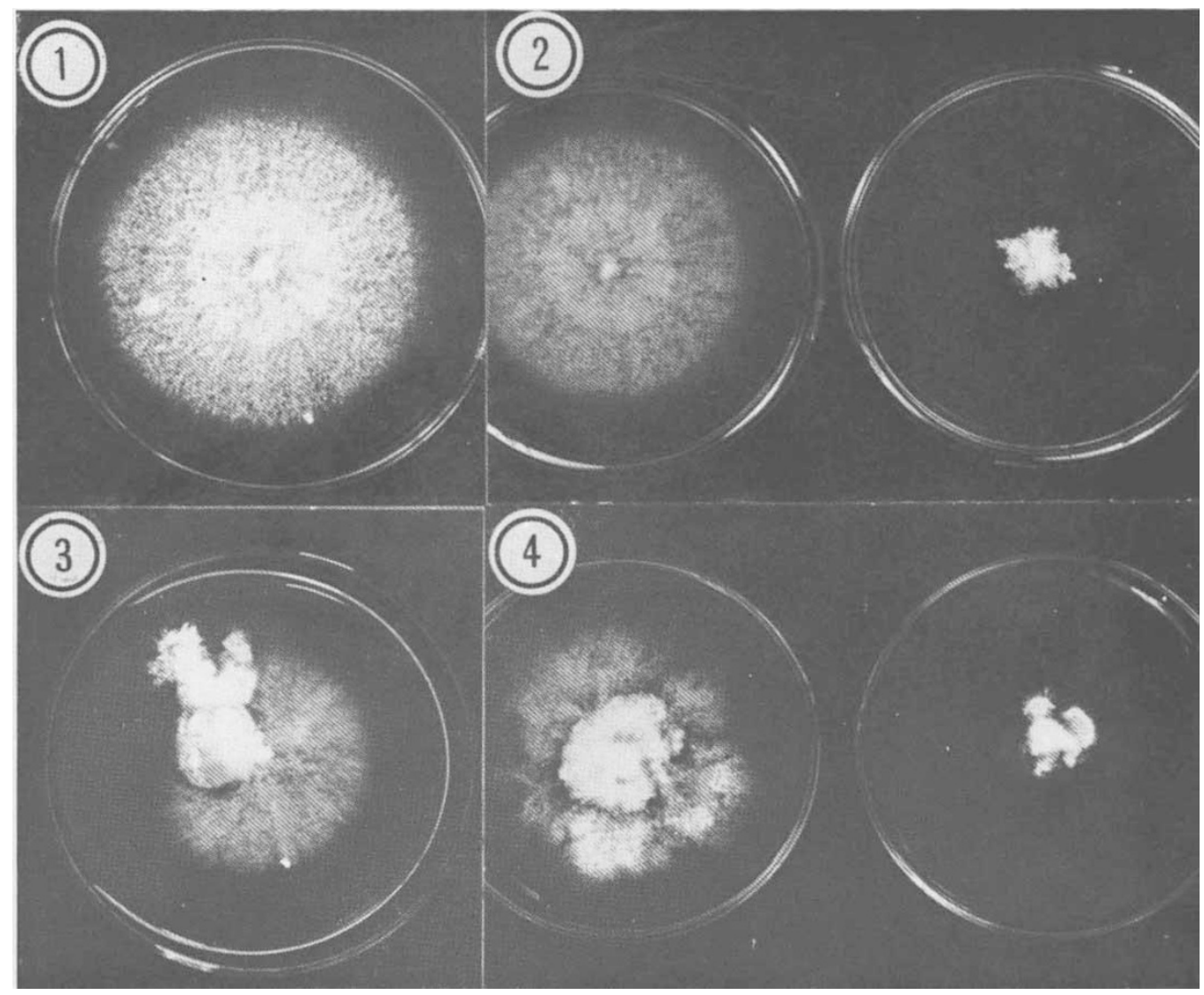

Plate I

Figs. 1-4.-Different types of mycelia resulting from mating $A 41 B 2 P$ (from experiment L7, table 2: B3016I, $A 41 B 2 P \times B 9020, A 42$ B51) with a tester $A 42$ B51. Pieces of pseudoclamped mycelia from above mating after being transferred to fresh plates developed pseudoclamped mycelia (fig. 1; fig. 2, left), dikaryotic mycelia (fig. 2, right; fig. 4, right) and dikaryotic mycelia with pseudoclamped sectors (fig. 3; fig. 4, left). 


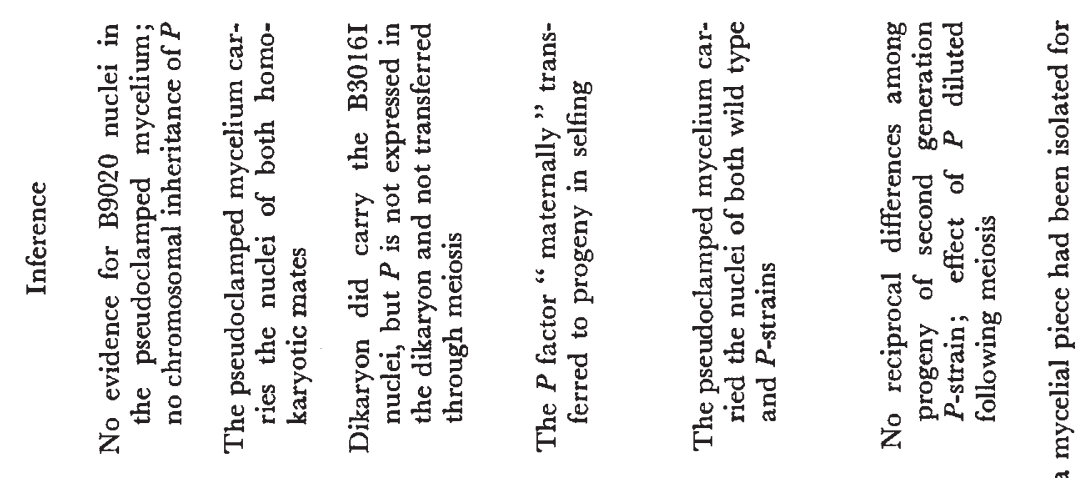

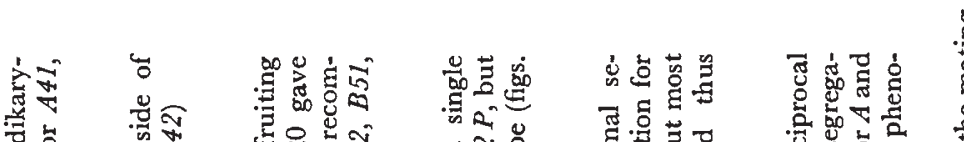

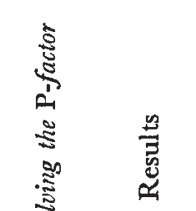

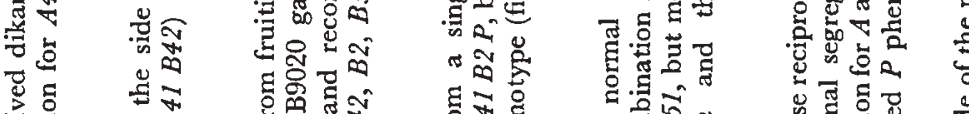

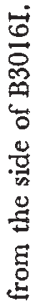

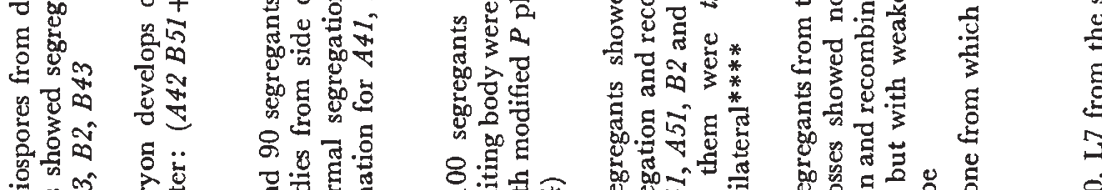

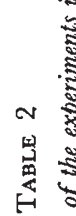

离

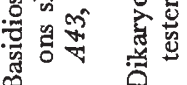

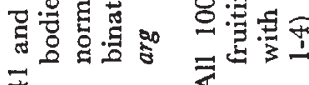<smiles>[X]C1=[C+]CC=[GeH]1</smiles>

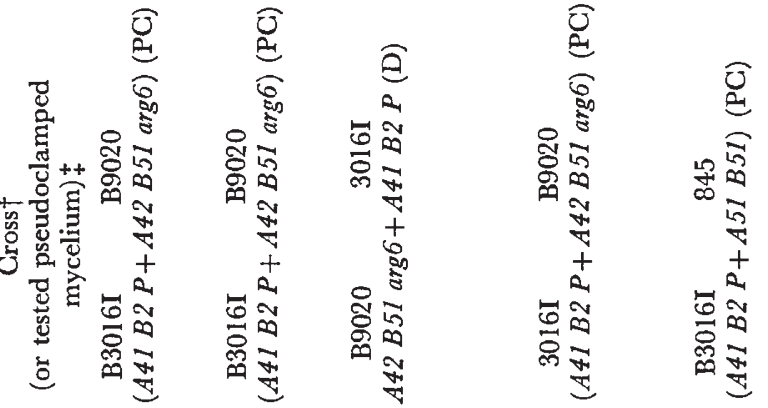

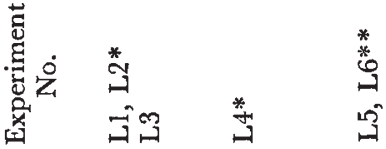

$\stackrel{*}{\stackrel{*}{*}}$ 吕

$\stackrel{\infty}{9}$

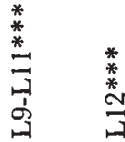

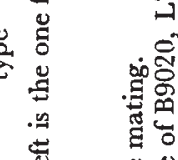
帘

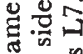
ㄹ⿺ㅇ . छ

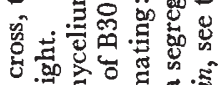

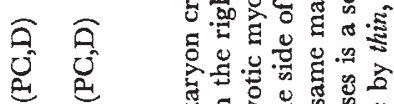

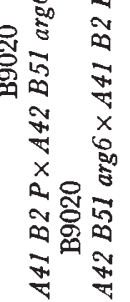

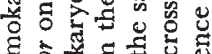
ฐ Хดी ธิ ‥

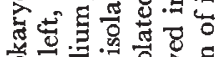

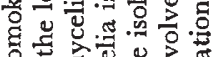

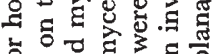

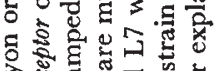

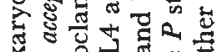

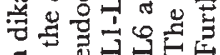

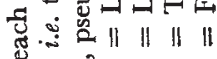

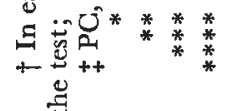




\section{(v) Modification of effect of $\mathrm{P}$ on mating reactions in following generations}

The segregants from the cross in experiment L7 showed a decreased effect of $P$ that did not always give asymmetrical matings. When these segregants were mated with three different testers, the mating reactions were as follows:

(a) With B9020 (A42 B51) all gave dikaryons, but with clear sectors of mycelia carrying pseudoclamps, on either or both sides of the mating. Transfers from the pseudoclamped sectors gave dikaryons, or pseudoclamped mycelia, or dikaryons with pseudoclamped sectors (figs. 1-4). The same happened to transfers from dikaryotic sectors.

(b) With L328 (A42 B2), the result was either a homokaryon or a pseudoclamped mycelium. The latter appeared predominantly on the side of the tested segregants, but also occasionally on the side of the tester.

(c) With 699 ( $A 41 B 41)$ the non-reciprocality was more pronounced; clear flat morphology appeared on the side of 699, pseudoclamps on the side of the $P$ segregants.

From class $(a)$ above, four fruiting bodies were further analysed, three from the side of the $P$ strain, one from the side of the normal partner (table 2, L9-L11 and L12). All the segregants behaved according to their $A$ and $B$ constitution, but the dikaryotisation was clearly slower on the side of the segregants in compatible matings. When the tester was already dikaryotised, regions with pseudoclamps as well as regions with true clamps appeared on the side of the segregant. Later, however, the side of the segregant was completely dikaryotised. Similarly, the flat morphology appeared late on the side of the segregants in common- $A$ combinations; a transition stage was observed in which the segregants were predominantly with pseudoclamps. It thus appears that the $P$ strain did not transfer to its progeny the clear-cut trait of asymmetric mating reaction. The segregants of all the four crosses, regardless of whether the $P$ strains served as "females" or " males", apparently carried what might be considered as remnants of the previously unilateral trait, showing as a transitional pseudoclamped stage appearing on the side of the segregants.

Unfortunately, the original $P$ strains lost their flat or homokaryotic morphology and all now carry pseudoclamps. Efforts to reisolate $P$ strains with normal morphology by hyphal-tip transfers were unsuccessful.

\section{The Gytoplasmic $F$ fagtor}

During a search for mutations in the $B$ factor, another extrachromosomal factor affecting mating reactions has been detected. A common- $B$ heterokaryon $(A \alpha 2-\beta 6 B 13+A \alpha 3-\beta 5 B 13)$ was treated with N-methyl-N-nitroso$\mathrm{N}^{\prime}$-nitroguanine (NG) (Parag et al., 1971), and many apparently dikaryotic spots were found and isolated (table 3). The presumed dikaryons failed to fruit, and one of them was di-mon mated (Papazian, 1950; Parag, 1962b) with Es-84, $A \alpha 4-\beta 1 B \alpha 1-\beta 2$. As would be expected of mutations affecting the $B$ factor specificity (Parag, 1962a), some of the segregants were flat (table 4). As no dikaryotic segregants were observed, and the spores were collected with a special device under the compound microscope (Raper, 1963), it is unlikely that the flat segregants resulted from double spores or disomics (Raper and Oetinger, 1962). These flat segregants were crossed with 
$A 97 B \alpha 6-\beta 7$ (table 5). If the conversion of the common- $B$ heterokaryon into a dikaryon and the appearance of flat segregants thereof, is a result of a chromosomal mutation, such a cross should lead to two possible results:

(a) If the mutation occurred in the $B$ factor, no $B 13$ segregants should be recovered, but only $B$ mutants and the $B$ of the tester $(B \alpha 6-\beta 7)$.

TABLE 3

A schematic representation of the steps in induction of $\mathrm{F}$ factor and the crosses carried to test the new mating specificity

\begin{tabular}{|c|c|c|c|}
\hline \multicolumn{4}{|c|}{ 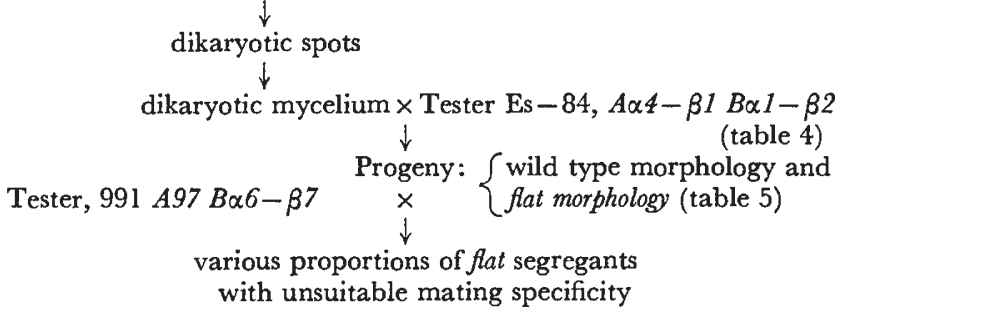 } \\
\hline \multicolumn{4}{|c|}{$\begin{array}{l}\text { Analysis of segregants from the di-mon mating } \\
((\mathrm{A} \alpha 2-\beta 6 \mathrm{~B} 13+\mathrm{A} \alpha 3-\beta 5 \mathrm{~B} 13) \mathrm{Bmut}) \times \mathrm{A} \alpha 4-\beta 1 \mathrm{~B} \alpha 1-\beta 2 *\end{array}$} \\
\hline Morphology & $\begin{array}{c}\text { ES }-84 \\
A \propto 4-\beta 1 B \alpha 1-\beta 2\end{array}$ & $\begin{array}{c}\text { Testers } \\
534-40 \\
A \alpha 2-\beta 6 B 13\end{array}$ & $\begin{array}{c}\text { MS }-7 \\
A \alpha 3-\beta 5 B \alpha 2-f\end{array}$ \\
\hline $\begin{array}{l}\text { Flat } \\
\text { Flat } \\
\text { Flat } \\
\text { Wild type }\end{array}$ & $\begin{array}{l}+ \\
F \\
F \&+ \\
+\end{array}$ & $\begin{array}{l}+ \\
+ \\
+ \\
-\end{array}$ & + \\
\hline Wild type & - & + & + \\
\hline Wild type & $F$ & & \\
\hline
\end{tabular}

The spores for this test were collected from the cross between the tester $(A \alpha 4-\beta 1$ $B \alpha 1-\beta 2)$ and the dikaryotic spot which resulted from the treatment of the stable common- $B$ heterokaryon $(A \alpha 2-\beta 6 B 13+A \alpha 3-\beta 5 B 13)$ with the mutagen NG.

(b) Or if the mutation is in a $B$ modifier, a quarter of the segregants should be $B 13$, and a half of the segregants should be flat and stable (Parag, $1962 a$; Raper and Raper, 1968). Seven crosses involving such flat segregants (table 5) showed significant deviations from one : one segregation for flat, and the analysis showed that none of the tested flat strains carried $B$ mutations. More significantly, these seven strains showed (table 4) mating reactions different from those expected of their genotypes as revealed in table 5 . In a cross between two flat strains, one would expect to find only flat segregants; however, such a cross $(28 \times 8$ in table 5$)$ gave no flat progeny. Following 2 months in stock, all the nine flat strains returned to normal (in morphology); four of them were retested and they showed the mating 
reactions expected of their genotypes, unlike the previous tests as shown in table 4 .

TABLE 5

Analysis of crosses involving the flat progeny from table 4

\begin{tabular}{|c|c|c|c|c|c|}
\hline $\mathrm{Cr}$ & & & & & Conclusions: \\
\hline $\begin{array}{l}\text { Flat Spor } \\
\text { from table }\end{array}$ & $\times$ Strain & $\begin{array}{l}\text { No. of spores } \\
\text { collected }\end{array}$ & $\begin{array}{l}\text { No. of } f \text { lat } \\
\text { spores }\end{array}$ & $\begin{array}{l}\text { factors in w.t. } \\
\text { progeny }\end{array}$ & $\begin{array}{l}\text { tested flat } \\
\text { strains }\end{array}$ \\
\hline No. 3 & 991 & 36 & 32 & $\begin{array}{l}B \alpha 1-\beta 2, B \propto 6-\beta 7 \\
A \propto 4-\beta 1\end{array}$ & $A \alpha 4-\beta 1 B \alpha 1-\beta 2$ \\
\hline No. 4 & 991 & 32 & 19 & $\begin{array}{l}B \propto 1-\beta 2, B \alpha 6-\beta 7 \\
A 97, A \propto 4-\beta 1\end{array}$ & $A \propto 4-\beta 1 \quad B \propto 1-\beta 2$ \\
\hline No. 7 & 991 & 21 & 0 & $\begin{array}{l}B \alpha 1-\beta 2, B \alpha 6-\beta 7 \\
A \alpha 2-\beta 6\end{array}$ & $A \alpha 2-\beta 6 B \alpha 1-\beta 2$ \\
\hline No. 11 & 991 & 42 & 0 & $\begin{array}{l}B \alpha 1-\beta 2, B \alpha 6-\beta 7 \\
A \alpha 2-\beta 6, A 97\end{array}$ & $A \alpha 2-\beta 6 B \alpha 1-\beta 2$ \\
\hline No. 5 & 991 & 31 & 0 & $\begin{array}{l}B \alpha 6-\beta 7 \\
A 97, A \alpha 4-\beta 1\end{array}$ & $A \alpha 4-\beta 1 B ?$ \\
\hline No. 13 & 991 & 29 & 2 & $\begin{array}{l}B \alpha 6-\beta 7 \\
A \alpha 4-\beta 1\end{array}$ & $A \propto 4-\beta 1 B \propto 1-\beta 2$ \\
\hline No. 13 & $534-40$ & 33 & 1 & $\begin{array}{l}B \propto 1-\beta 2, B 13 \\
A \propto 4-\beta 1, A \propto 2-\beta 6\end{array}$ & $A \propto 4-\beta 1 B \propto 1-\beta 2$ \\
\hline No. 28 & $\begin{array}{l}\text { Flat } \\
\text { segregant } \\
\text { No. } 8\end{array}$ & $250^{*}$ & 0 & $\begin{array}{l}B \alpha 1-\beta 2, B 13 \\
A \propto 4-\beta 1, A \propto 2-\beta 6\end{array}$ & \\
\hline
\end{tabular}

* Only 50 analysed for mating type factors.

\section{Discussion}

Jinks (1963) described several examples of extrachromosomal inheritance in fungi. These results seem to add two more examples, this time suggesting evidence for extrachromosomal factors that control mating reactions and mimic genetically controlled heterokaryosis.

One technical obstacle halted the study of the $P$ factor. The three strains, B3006I, B3006III, B3016I, kept in $c a .5^{\circ} \mathrm{C}$, started to carry pseudoclamps without being mated. In addition to making a further genetic analysis impracticable, this change seemed to call into question the asymmetrically formed pseudoclamped mycelia as previously observed. It could be assumed that these specific strains carry a chromosomal gene controlling the formation of pseudoclamps in the homokaryon, and these were the pseudoclamps that appeared on the side of the $P$ strains in every mating. However, in parallel with every mating, the $P$ strains used as controls did not show pseudoclamps. Moreover, results of several crosses refuted such an interpretation. The mycelium formed in experiment L8 (table-2) was clearly ( $A 41 B 2 P+A 51 B 51$ ), and thus should have been dikaryotic with true clamp connections. The progeny of six crosses involving $P$ (table 2, L1-L3, L5-L7) did not show any pseudoclamps as homokaryons, which means that the parental $P$ homokaryon did not carry a chromosomal gene which causes pseudoclamp formation in the homokaryon.

The theory of an extrachromosomal modifier epistatic to $A$ and $B$ factors seems to agree with the results. The $P$ factor determines the appearance of pseudoclamps in any heterokaryon carrying it in one of its nuclear components, schematically:

$$
(A x B x P+A y B y) \rightarrow \text { pseudoclamps. }
$$


This factor does not migrate from one partner of the mating to the other partner as the nuclei do, and this explains the asymmetrical results: in the presence of two types of nuclei the $P$ factor in the cytoplasm induces the formation of pseudoclamps. Apparently not all the cells on the side of the $P$ strain also carry the nuclei of the other strain, as in some of the crosses these nuclei were not recovered (table 2, L7). This is not surprising, since selfedfruiting bodies had been found on pseudoclamped common- $B$ heterokaryons (Parag, 1960), and predominance of uninucleate cells in pseudoclamped mycelia is predicted from the mode of nuclear divisions as well as observed in few experiments (Parag, 1964, 1965, 1970). This interpretation explains why $P$ does not appear among segregants of fruiting bodies appearing on the dikaryons developed on the side of the mating partner that does not carry the $P$ factor in its cytoplasm (table 2, L5, L6). It can be transferred via meiosis of the fruiting bodies carried the $P$ factor in their cytoplasm, as did happen in the selfed fruiting body (table 2, L7). The effect of the $P$ factor clearly changed, however, following meiosis, and was now fully expressed only in common- $A$ matings. The appearance of pseudoclamps after long preservation of the stain B3006I and B3016I can also be explained. A genetic change probably occurred, with the $P$ factor lost from part of the cells, and the result might have been a heterokaryon $(A 41 B 2 P+A 41 B 2)$, which on the basis of previous observations should carry pseudoclamps, although it carries only one $A$ and one $B$.

In matings in Schizophyllum commune, heteroallelism in the $A$ factor switches on the initiation of pseudoclamps; heteroallelism in the $B$ factor switches on the completion of clamp connections (Parag, 1965; Raper and Raper, 1968). The $P$ factor apparently leads to a bypass of this control by the incompatibility factors; it does not needs the "switch on" signal of $A$ in order to initiate the pseudoclamps and it ignores the "switch on" signal of $B$ to complete the clamp connections. It thus has effects on the constitutivity of the developmental stages of the dikaryon similar to the chromosomal modifiers (Raper and Raper, 1968). In view of the recent publications of correlation between virus-like particles and cytoplasmic inheritance in $S$. commune (Koltin et al., 1973) it would be interesting to do electron microscope examinations of the pseudoclamped strains carrying the $P$ factor.

From the crosses involving the $F$ factor, it has been shown that the results cannot be explained on the basis of chromosomal factors, neither mutations in the $B$ factor, nor $B$ modifiers. It appears that an extrachromosomal change occurred following the NG treatment, causing the progeny with this change to show mating-type reactions which do not fit their genotypes; this change also caused the flat morphology as expected of a loss of $B$ specificity (Parag, 1962a). These changes were inherited in a non-Mendelian fashion (table 5), and showed an extreme instability. When, as the result of this instability the strains lost the $F$ factor, they regained both the correct mating specificities, as expected from their genotypes, and the normal morphology.

The effect of the $P$ factor revealed an additional genetic entity that controls the sequence of events leading to the dikaryon. It is very probable, at least by the mode of operation in the two sides of the matings, that it is extranuclear. The discovery of such a factor, or better to say the way by which it can be recognised, emphasises the usefulness of the Basidiomycetes as organisms for investigating nucleo-cytoplasmatic relationships. Following the matings, nuclei of a certain genotype migrate into a cytoplasm previously 
controlled by another genotype. This transition period represents a mass "transplantation" of nuclei into a foreign cytoplasm. It is clearly seen in ordinary compatible matings that freshly established dikaryons on each side of the confrontation line are morphologically different, and it takes some time of growth as established dikaryons to erase these differences. On the other hand, dikaryons can be dedikaryotised, and a transitional state develops in which one nucleus is embedded in a cytoplasm previously controlled by two nuclei. An ingenious experiment of the latter type was done by microsurgery by Harder (1927). To keep open eyes and mind on compatible matings in Basidiomycetes as tools for investigating nucleuscytoplasm relationship might be worthwhile.

Acknowledgments.-The author thanks Dr M. Grindle for the critical reading of the manuscript and $\mathrm{Mr} \mathrm{M}$. Shalev for his skilled participation in the research on the $F$ factor. This research was supported by grant No. A10-CR-66 of the U.S. Department of Agriculture.

\section{REFERENCES}

HARDER, R. 1927. Zur Frage nach der Rolle von Kern und Protoplasma in Zellgeschehen und bei der übertragung von Eigenschaften. Z. Bot., 19, 337-407.

JINKs, J. L. 1963. Cytoplasmic inheritance in fungi. In: Burdette, W. J. (ed)., Methodology in Basic Genetics, pp. 325-354. Holden-Day, San Francisco.

KOLTIN, Y., BERICK, R., STAMBERG, J., AND BEN-SHAUL, Y. 1973. Virus-like particles and cytoplasmatic inheritance of plaques in a higher fungus. Nature, New Biol., 241, 108109.

Papazian, H. P. 1950. Physiology of the incompatibility factors in Schizophyllum commune. Bot. Gaz., 112, 143-163.

PARAG, y. 1960. Genetic Studies on Somatic Recombination and Common-B Heterokaryosis in Schizophyllum commune. Ph.D. Thesis, Harvard University.

PARAG, y. 1962a. Mutations in the $B$ incompatibility factor in Schizophyllum commune. Proc. Nat. Acad. Sci., U.S.A., 48, 743-750.

PARAG, Y. 1962b. Studies on somatic recombination in dikaryons of Schizophyllum commune. Heredity, 17, 305-318.

PARAG, Y. 1964. Diploid mycelia resulting from common- $B$ matings of Schizophyllum commune. Abst. 10th Intern. Botan. Congr., Edinburgh, pp. 441-442.

PARAG, Y. 1965. Common-B heterokaryosis and fruiting in Schizophyllum commune. Mycologia, $57,543-561$.

PARAG, Y. 1970. Genetics of Tetrapolar Sexuality in Higher Fungi: The B-factor, common-B Heterokaryosis and Parasexuality. U.S. Department of Agriculture, Final Report, Jerusalem.

PARAG, Y., BEN-ShaUl, R., AND LAVIE, B. 1971. Dominance and non-complementation among pink-adenineless mutants of Schizophyllum commune involving two discrete genes. Molec. Gen. Genet., 113, 345-354.

PARAG, Y., AND NAGHMAN, B. 1966. Diploidy in the tetrapolar heterothallic basidiomycete. Schizophyllum commune. Heredity, 21, 151-154.

RAPER, J. R. 1963. Device for the isolation of spores. F. Bacteriol., 86, 342-344.

RAPER, J. R. 1966. Genetics of Sexuality in Higher Fungi. Ronald Press, New York.

RAPER, J. R., BOYD, D. H., AND RAPER, C. A. 1965. Primary and secondary mutations at the incompatibility loci in Schizophyllum commune. Proc. Nat. Acad. Sci., U.S.A., 53, 1324-1332.

RAPER, J. R., KRONGELB, G. S., AND BAXTER, M. G. 1958. The number and distribution of incompatibility factors in Schizophyllum commune. Amer. Nat., 92, 221-232.

RAPER, J. R., AND MILES, P. G. 1958. The genetics of Schizophyllum commune. Genetics, 73, 530-546.

RAPER, J. R., AND OETINGER, M. T. 1962. Anomalous segregation of incompatibility factors in Schizophyllum commune. Revta. Biol. Lisb., 3, 205-221.

RAPER, J. R., AND RAPER, C. A. 1968 . Genetic regulation of sexual morphogenesis in Schizophyllum commune. J. Elisha Mitchell Sci. Soc., 84, 266-273. 